\title{
Mobile Base Stations Placement and Energy Aware Routing in Wireless Sensor Networks
}

\author{
A. P. Azad and A. Chockalingam \\ Department of ECE, Indian Institute of Science, Bangalore 560012, India
}

\begin{abstract}
Increasing network lifetime is important in wireless sensor/ad-hoc networks. In this paper, we are concerned with algorithms to increase network lifetime and amount of data delivered during the lifetime by deploying multiple mobile base stations in the sensor network field. Specifically, we allow multiple mobile base stations to be deployed along the periphery of the sensor network field and develop algorithms to dynamically choose the locations of these base stations so as to improve network lifetime. We propose energy efficient low-complexity algorithms to determine the locations of the base stations; they include $i$ ) Top- $K_{\max }$ algorithm, $i i$ ) maximizing the minimum

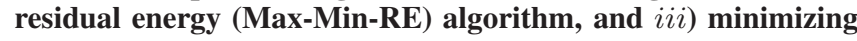
the residual energy difference (MinDiff-RE) algorithm. We show that the proposed base stations placement algorithms provide increased network lifetimes and amount of data delivered during the network lifetime compared to single base station scenario as well as multiple static base stations scenario, and close to those obtained by solving an integer linear program (ILP) to determine the locations of the mobile base stations. We also investigate the lifetime gain when an energy aware routing protocol is employed along with multiple base stations.
\end{abstract}

Keywords - Multiple mobile base stations, network lifetime, energy efficiency

\section{INTRODUCTION}

A key issue in wireless sensor/ad-hoc networks is maximizing the network lifetime and the amount of useful data successfully transferred during the network lifetime. In sensor networks, the data transport model is such that a base station, typically located at the boundary of or beyond the field from where the sensors sense/measure data, collects data from the sensors which are distributed in a field from where data measurement/collection has to be performed [1]-[6]. Typically, the sensor nodes, in addition to behaving as source nodes in generating data to be passed on to the base station, act as intermediate relay nodes as well to relay data from other source nodes towards the base station on a multihop basis. Therefore, while energy will be spent by a node in receiving and forwarding all transit packets, energy thus spent may not add to the end-to-end delivery of the packet (i.e., packets may still have more hops to reach the base station). This can result in reduced efficiency, in terms of total amount of data delivered to the base station per joule of energy in the network, particularly when the hop length between the source node(s) and base station gets larger. This problem can be alleviated by the use of multiple base stations deployed along the periphery of the field, and allowing each base station to act as a data sink [7]. That is, each sensor node can send its data to any one of these base stations (may be to the base station towards which the cost is minimum). Base stations can communicate

This work was supported in part by the Indo-French Centre for Promotion of Advanced Research, New Delhi, under Project 2900-IT. among themselves to collate the data collected (energy is not a major concern in the communication between the base stations). Deploying such multiple base stations essentially can reduce the average hop length between the source-sink pairs, thus enabling to achieve increased network lifetime and larger amount of data delivered during the network lifetime.

A relevant question in the above multiple base station approach is how to determine optimum locations of these base stations that will maximize the network lifetime. Our focus in this paper is to address this question. We consider a set of feasible base station locations (sites) on the boundary of the sensor field. Given that $K, K \geq 1$ base stations can be deployed, the problem to solve is to choose the optimum locations for these $K$ base stations from the set of feasible sites. We divide the time axis into rounds of equal period. Placement of base stations is carried out at the beginning of each round and held for the entire duration of the round. A new placement is carried out in the beginning of the next round, and so on, till the end of network life. In [7], the base stations placement problem is formulated as an optimization problem and the optimum base stations locations are obtained as the solution to an Integer Linear Program. The complexity of the ILP solution however is high. Here, we propose energy efficient low-complexity algorithms to determine the base station locations; they include $i$ ) Top- $K_{\max }$ algorithm, ii) maximizing the minimum residual energy (Max-Min-RE) algorithm, and $i i i)$ minimizing the residual energy difference (MinDiff-RE) algorithm. We show that the proposed base stations placement algorithms provide increased network lifetimes and amount of data delivered during the network lifetime. We also investigate the lifetime gain when an energy aware routing protocol is employed along with multiple base stations.

The rest of the paper is organized as follows. In Section II, we present the system model. In Section III, we present the proposed base stations placement algorithms. In Section IV, we present the performance results and discussions. Finally, conclusions are given in Section V.

\section{System Model}

We consider the following system model and assumptions.

A. Network: A set of sensor nodes $V_{s}$ are uniformly distributed over a square sensor field. A set of feasible sites $V_{f}$ (i.e., feasible base station locations) along the periphery of the sensor field is assumed. The sensor network is then represented as a graph $G(V, E)$, where $V=V_{s} \cup V_{f}$, and $E \subseteq V \times V$ represents the set of wireless links. Wireless links 
between sensor nodes and a feasible site refer to the links that would exist if a base station is located at that particular site.

There are $K, K \geq 1$ base stations to deploy. Time is divided into rounds of equal period. Selection of base station locations from the set of feasible sites $V_{f}$ is made at the beginning of each round, and the base stations are moved and placed in these chosen locations for the entire duration of that round. A new set of base station locations are computed at the beginning of the next round (and the base stations moved to these newly chosen locations), and so on, till the end of network life.

B. Transceiver: The transmission range of all sensor nodes is same and fixed. As in similar system models in the literature [1], the energy spent in transmitting a bit over a distance $d$ is assumed to be proportional to $d^{2}$.

C. MAC and Routing: Data packets generated at each sensor node is assumed to be of equal length. Also, each sensor node is assumed to generate equal amount of data per unit time. At the MAC level, Self-organizing Medium Access Control for Sensor networks (SMACS), a contention-free MAC protocol presented in [8] is employed to provide channel access for all the sensor nodes. For multi-hop routing, minimum cost forwarding (MCF) protocol presented in [9] is employed.

D. Battery and Network life: Each sensor node is powered by a finite-energy battery with an available energy of $E_{\text {battery }} \mathrm{J}$ at the initial network deployment. At the sensor node, transmission of a data packet consumes $E_{t} \mathrm{~J}$ of energy, and reception of a data packet consumes $E_{r} \mathrm{~J}$. A sensor node ceases to operate if its battery is drained below a certain energy threshold (i.e., available energy goes below a certain usable threshold). Often, network lifetime is defined as the time for the first node to die [1],[5] or as the time for a certain percentage of nodes to die [6]. Here, we define the network life time as follows. The end of network life is said to be reached either if the batteries of all the sensor nodes are drained below the usable threshold or if all the live sensor nodes are disconnected from all the feasible sites (i.e., either all nodes die or all live nodes are out of range of all feasible sites).

With the above system model, we need to choose optimum base station locations from $V_{f}$ at the beginning of each round. In the following section, we present efficient algorithms for this base station placement problem.

\section{Base Stations Placement Algorithms}

The base stations placement problem in the above system model has been formulated as an optimization problem in [7], and the optimum base station locations are obtained as the solution to an ILP. However, the complexity of the ILP solution is high. In this section, we propose three energy efficient low-complexity algorithms to solve the base stations placement (BSP) problem.

Let $s_{i}$ denote the location of sensor node $i, i \in V_{s}$, and $f_{i}$ denote the location of feasible site $i, i \in V_{f}$. Let $r$ denote the transmission range of each sensor node. Let $R E_{i}$ denote the residual battery energy in sensor node $i$ at the beginning of a round when the base station locations are computed.

\section{A. Top- $K_{\max }$ Algorithm}

This algorithm selects those feasible sites (maximum $K$ sites) whose nearest neighbour nodes have the highest residual energies. Since the first-hop neighbour node to a base station has to handle all the transit packets from other nodes towards that base station, it can drain its battery sooner. Therefore, by assigning the nearest neighbour with highest residual energy nodes to serve as the first-hop neighbour nodes in each round, the life of the nodes in the network and hence network lifetime can get extended. The algorithm works as follows.

1) For each feasible site $i \in V_{f}$, find the nearest sensor node $n_{i}$ within the connectivity range $r$, i.e., for each $i \in V_{f}$ choose sensor node $n_{i} \in V_{s}$ such that

$$
\left|f_{i}-s_{n_{i}}\right| \leq\left|f_{i}-s_{j}\right|, \forall j \in V_{s}, j \neq n_{i} .
$$

and

$$
\left|f_{i}-s_{n_{i}}\right| \leq r
$$

2) Order these nearest neighbour nodes $\left\{n_{i}, i \in V_{f}\right\}$ in descending order of their residual energies, $R E_{n_{i}}$.

3) Select a maximum of $K$ nodes from the top in this ordered list, and declare their corresponding nearest feasible sites as the solution.

As can be seen, this is a greedy algorithm. A main advantage of this algorithm is its simplicity and less computation complexity. If $n$ is the number of sensor nodes and $N$ is the number of feasible sites, then the complexity of this algorithm is given by $n N+N \log N$, which is linear in $n$.

\section{B. Max-Min-RE Algorithm}

Since the Top- $K_{\max }$ solution in the above algorithm gives preference to nearest neighbour nodes, it is likely that the nodes nearer to the feasible sites are loaded heavily and their batteries drained sooner than other nodes. The following MaxMin-RE algorithm (maximizing the minimum residual energy algorithm) attempts to distribute the load more evenly to different nodes. The Max-Min-RE algorithm works as follows. There are $N$ feasible sites and $K$ base stations to deploy, $N \geq K$. The number of base stations placement solutions are $P=\left(\begin{array}{l}N \\ K\end{array}\right)$. Let this solution set be $S$. Let the $j$ th solution in the solution set $S$ be denoted by $T_{j}$.

1) Determine set $S_{c} \subseteq S$ such that $S_{c}=\left\{T_{j}: \forall i \in V_{s}\right.$ there exists $p \in V_{s}$ such that $\left|s_{i}-s_{p}\right| \leq r$ or $q \in V_{f}$ such that $\left.\left|s_{i}-f_{q}\right| \leq r\right\}$.

2) For a given solution $T_{j} \in S_{c}$, determine the routes from all the sensor nodes to their respective base stations using MCF routing.

3) For each node $i \in V_{s}$ compute the energy consumed at all nodes in the path in delivering a data packet from node $i$ to its corresponding base station, and determine the resulting residual energies in all nodes.

4) Find the minimum residual energy among all nodes in the $j$ th solution

$$
M_{j}=\min _{i \in V_{s}}\left\{R E_{i}\right\}
$$




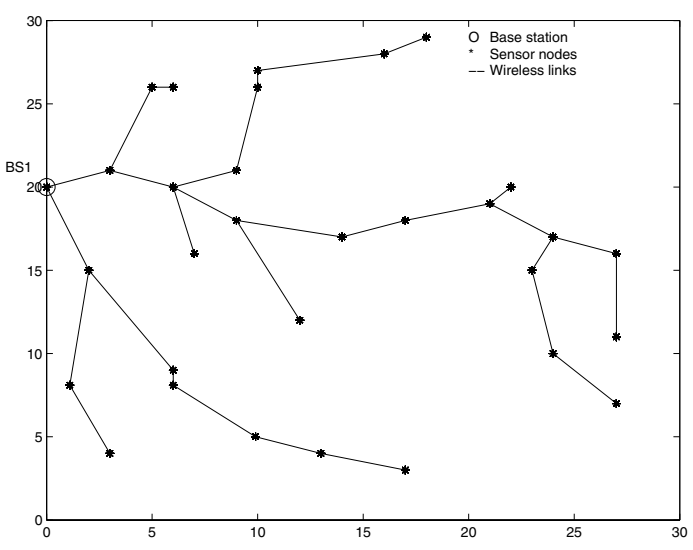

Fig. 1. MCF routing in single base station scenario.

5) Choose the solution as

$$
T_{\text {Max-Min-RE }}=\max _{j}\left\{M_{j}: T_{j} \in S_{c}\right\} .
$$

By this algorithm, we are choosing that solution in which the heavily loaded node (identified by the minimum residual energy among various nodes in a given solution, and not by the minimum distance as done in the Top- $K_{\max }$ algorithm) has the maximum residual energy among all possible solutions. This algorithm hence is expected to distribute (and drain energy) evenly among the nodes without bias to nearest neighbour nodes. The algorithm results in a complexity of $P\left(N n+K n+a n^{2}+N \log N+\log P\right)$, i.e., since $N, K$ and $P$ are typically small compared to $n$, the algorithm has $n^{2}$ complexity.

\section{MinDiff-RE Algorithm}

In this algorithm, which we call as MinDiff-RE algorithm (minimizing the difference in residual energy algorithm), the idea again is to evenly drain the nodes. It is similar to the Max-Min-RE algorithm, except that we choose that solution for which the difference between the maximum and minimum residual energies in nodes is minimized (rather than maximizing the minimum residual energy as in Max-Min-RE algorithm. The MinDiff-RE algorithm works as follows.

1) Perform steps 1) to 3) of the Max-Min-RE algorithm.

2) Compute the metric

$$
M_{j}=\max _{i \in V_{s}}\left\{R E_{i}\right\}-\min _{i \in V_{s}}\left\{R E_{i}\right\} .
$$

3) Choose the solution as

$$
T_{M i n D i f f-R E}=\min _{j}\left\{M_{j}: T_{j} \in S_{c}\right\} .
$$

The complexity of this algorithm is same as that of the MaxMin-RE algorithm presented in Sec. III-B.

\section{Performance Results}

We evaluated the performance of the above base stations placement (BSP) algorithms through simulations. The simulation model is as follows.

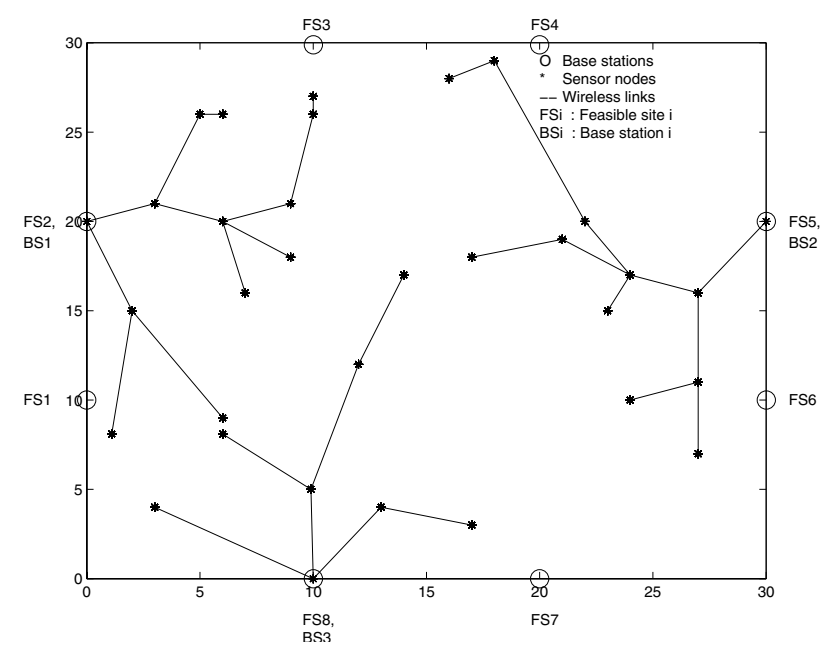

Fig. 2. MCF routing in three base stations scenario.

\section{A. Simulation Model}

A square sensor field of area $30 \mathrm{~m} \times 30 \mathrm{~m}$ (Fig. 1) is considered. The number of sensor nodes in the network is 30 . Sensor nodes are uniformly distributed in the network area. The number of feasible sites is taken to be 8 , and the coordinates of these feasible sites are $\{(0,10),(0,20),(10,30),(20,30)$, $(30,20),(30,10),(20,0),(10,0)\}$, as shown in Fig. 2. The maximum number of mobile base stations is taken to be 3 (i.e., $K=3$ ). Each sensor node is provided with an initial energy of $E_{\text {battery }}=0.05 \mathrm{~J}$. The transmission range of each sensor node is set to 10 meters. As in [1], the energy spent in transmitting a bit over 1 meter distance is taken as $0.1 n J / b i t-m^{2}[7],[1]$ and the energy spent in receiving a bit is set to $50 \mathrm{~nJ} / \mathrm{bit}$. The packet length is fixed at $200 \mathrm{bits}$. Each round lasts 300 time frames. Each node generates 1 packet every 30 frames; i.e., each node generates 10 packets per round. The performance measures of interest are the network lifetime (as defined in Sec. II.D) and the amount of data packets delivered during the lifetime of the network. We evaluate and compare the above performance measures for various BSP algorithms, including:

1) Single static base station

2) Three static base stations

3) Three mobile base stations, Top $K_{\max }$ algorithm

4) Three mobile base stations, Max-Min-RE algorithm

5) Three mobile base stations, MinDiff-RE algorithm

6) Three mobile base stations, ILP solution (this scheme is same as Scheme 4 in [7])

In scheme 1), only one base station is used in a fixed location (as shown in Fig. 1). In scheme 2, three base stations are used, but at fixed locations as shown in Fig. 2 (these locations are not changed from one round to the other). Schemes 3 ), 4) and 5) are the proposed Top- $K_{\max }$, Max-Min-RE, and MinDiff-RE algorithms, respectively. The case of ILP solution in scheme 6) corresponds to the optimization problem formulation presented in [7], which is stated as below: Let $y_{l}$ be a $0-1$ integer variable for each $l \in V_{f}$ such that $y_{l}=1$ if a base station is located at feasible site $l$; 0 otherwise. Defining $\mathcal{N}(i)$ to be the set of neighbours of node $i, L$ to be the 


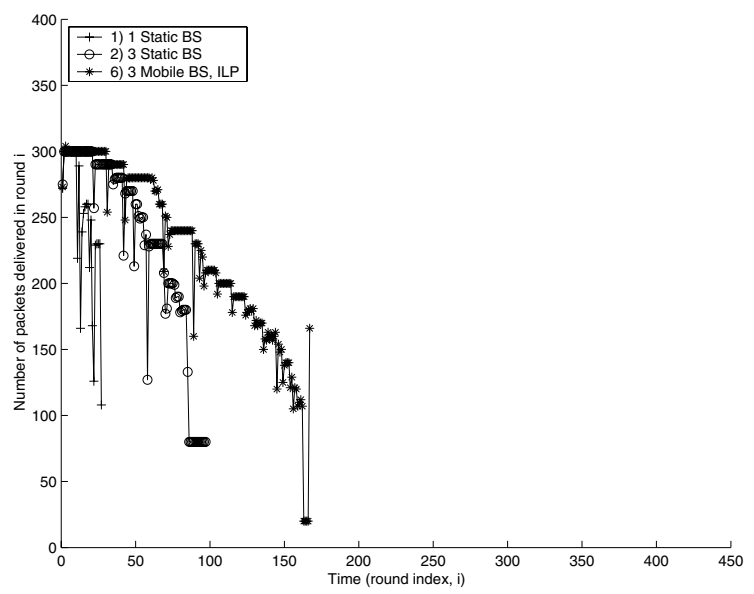

Fig. 3. Traces of number of packets delivered per round as a function of time for schemes 1), 2), and 6). MCF routing. Initial energy at each node, $E_{\text {battery }}=0.05 \mathrm{~J}$. One packet $=200$ bits. Range of each node, $r=10 \mathrm{~m}$.

number of time frames per round, $x_{i j}$ to be the number of packets node $i$ transmits to $j, j \in \mathcal{N}(i)$, and $0<\alpha \leq 1$, the solution $y_{l}, l \in V_{f}$ is obtained by solving the following ILP which minimizes the maximum energy spent, $E_{\max }$, by a sensor node in a round

$$
\begin{gathered}
\text { Minimize } E_{\max } \\
\sum_{j \in \mathcal{N}(i)} x_{i j}-\sum_{k \in \mathcal{N}(i)} x_{k i}=L, i \in V_{s} \\
E_{t} \sum_{j \in \mathcal{N}(i)} x_{i j}+E r \sum_{k \in \mathcal{N}(i)} x_{k i} \leq \alpha R E_{i}, i \in V \\
\sum_{i \in V_{s}} y_{l} \leq K \\
E_{t} x_{i k} \leq L\left|V_{s}\right| y_{k}, \quad k \in V_{f} \\
x_{i j} x_{i j}+E_{r} \sum_{k \in \mathcal{N}(i)} x_{k i} \leq E_{\max }, i \in V \\
i \in V_{s}, j \in V ; y_{k} \in\{0,1\}, k \in V_{f} .
\end{gathered}
$$

We have used CPLEX (version 9) to solve the above ILP. As in [7], for each instance, the value of the parameter $\alpha$ was initially set to 0.2 and incremented in steps of 0.2 in case the instance was infeasible.

\section{B. Results and Discussions}

We simulated the six different BSP schemes in the above and evaluated their relative performance in terms of network lifetime and amount of data delivered during the network lifetime. First, we illustrate the number of packets delivered to the base station(s) in a round as a function of time (measured in number of rounds) for a given realization of the distribution of the sensor nodes. Figures 3 and 4 show this behaviour of number of packets delivered in a round over time; Fig. 3 shows the traces for schemes 1), 2) and 6), and Fig. 4 shows the traces for the proposed schemes 3), 4) and 5).

From Fig. 3, it can be seen that the network life ends at the 27 th round itself in the case of single static base station

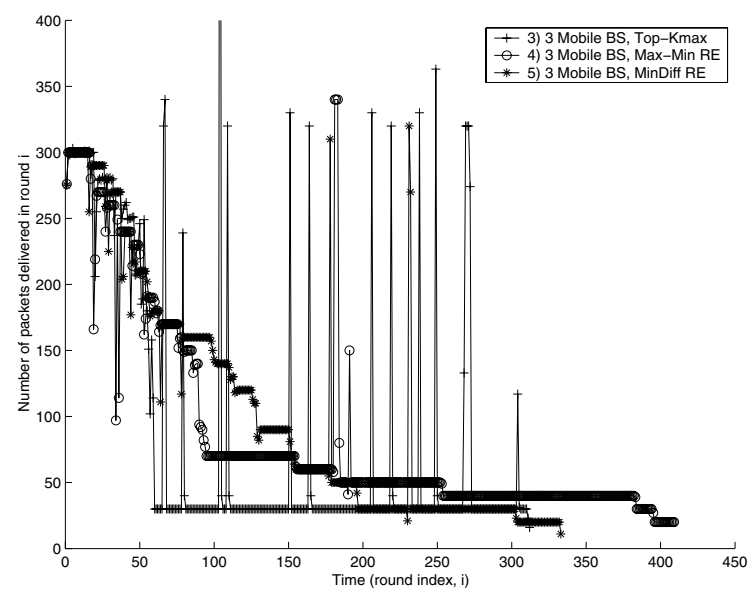

Fig. 4. Traces of number of packets delivered per round as a function of time for the proposed schemes 3), 4), and 5). MCF routing. Initial energy at each node, $E_{\text {battery }}=0.05$ J. One packet $=200$ bits. Range of each node, $r=10 \mathrm{~m}$.

(scheme 1), whereas with three static base stations (scheme 2) the network life extends to 97 rounds. This is expected since, as we pointed out earlier, in the one base station case, the average hop length required to deliver a packet to the base station (and hence the energy spent) is more compared to the three static base stations case. The network life is further extended to 167 rounds when mobile base stations are deployed at the locations solved by the ILP at the beginning of each round (scheme 6). Also, it is seen that the number of packets delivered in a round decreases as the round index (i.e., time) increases. This is because when nodes expire (indicated by the round indices where sharp fall and immediate rise of number of packets delivered are seen), the total rate of generation of new packets in the network reduces which reduces the maximum number of packets delivered in a round. The sharp fall in the number of packets delivered in a round is attributed to a node expiring in the middle of a round (typically, this node could be the first hop node to a base station), the expiry of which stops packet delivery to the base station till the end of that round. The number of packets delivered rises sharply in the very next round since a new set of base station locations are found (in case of scheme 6 ) and the routing gets updated (in all the schemes 1,2, and 6) at the beginning of the very next round. It can be further noted that the area under the trace for a given BSP scheme gives the total number of packets delivered in the network lifetime in that scheme.

From the traces in Figs. 3 and 4 it can be seen that the time for the first node to die is the least in the single base station case (scheme 1) and the most in the ILP case (scheme 6). However, the lifetime till the last node dies is larger in the proposed schemes. For example, in Fig. 4, we observe that the network lifetime achieved by the proposed BSP algorithms are significantly higher; 312 rounds for Top- $K_{\max }$ algorithm (scheme 3), 365 rounds for Max-Min-RE algorithm (scheme 4), and 380 rounds for Min-Diff-RE algorithm (scheme 5). The high variability of the number of packets delivered in a round for the Top- $K_{\max }$ algorithm (mainly after round 60 in Fig. 4) can be attributed to the following: many of the 'close' neighbours of the feasible sites expire before the 60th round, 


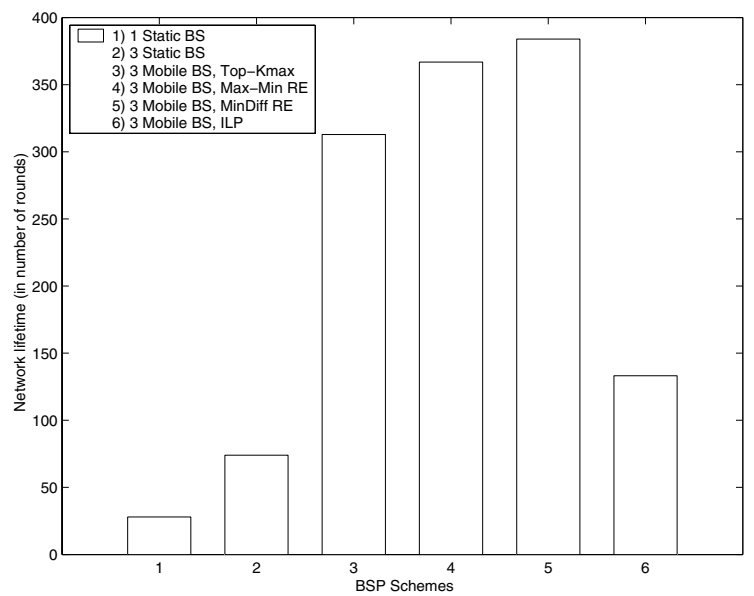

Fig. 5. Network lifetime in number of rounds for different BSP algorithms. $\mathrm{MCF}$ routing. Initial energy at each node, $E_{\text {battery }}=0.05 \mathrm{~J}$. One packet $=$ 200 bits. Range of each node, $r=10 \mathrm{~m}$.

during which time other nodes buffer many transit packets, and, when these nodes get selected as the first-hop neighbour node in the next rounds, all these buffered packets get delivered (which in turn consumes large amount of energy that can make the node expire) resulting in the sharp peaks. The variation of the number of packets delivered per round is more smooth in the Min-Max-RE and MinDiff-RE algorithms. The long tail in the traces of Max-Min-RE and MinDiff-RE algorithms indicate the possibility of just a few (typically one or two) nodes remaining alive in the network within the connectivity range of the feasible site(s); these nodes need much less energy since they need to transmit mainly their own packets (and very few transit packets from other nodes) because of which they survive longer keeping the network alive.

Next, in Fig. 5, we illustrate the average network lifetime performance of the various BSP algorithms. The network lifetimes for various BSP schemes shown in Fig. 5 are the simulated lifetimes averaged over 100 independent realizations of the distribution of the locations of the sensor nodes. For the same simulation runs, Fig. 6 shows the amount of data delivered during the network lifetime for the different BSP algorithms. The $95 \%$ confidence interval for the simulation results plotted in Figs. 5 and 6 are tabulated in Table I. From Figs. 5 and 6, it can be seen that the proposed BSP algorithms (schemes $3,4,5$ ) perform better than the single base station scheme (scheme 1) and fixed three base station scheme (scheme 2). Among the proposed schemes, scheme 4 (Minmax-RE) and scheme 5 (MinDiff-RE) perform best, achieving highest network lifetimes (about 360 rounds) and largest amount of data delivered (about $3.5 \times 10^{4}$ packets). The proposed schemes 4 and 5 give longer lifetimes than scheme 6 since the proposed schemes effectively use the residual energy information of the various nodes.

The total available energy in the entire network is $1.5 \mathrm{~J}$ (i.e., 30 nodes each having an initial energy of $0.05 \mathrm{~J}$ ). It would be of interest to know how this total available energy in the network has been utilized by the different BSP algorithms. Toward this end, in Fig. 7, we plot the total energy spent by different algorithms over the entire network lifetime (averaged

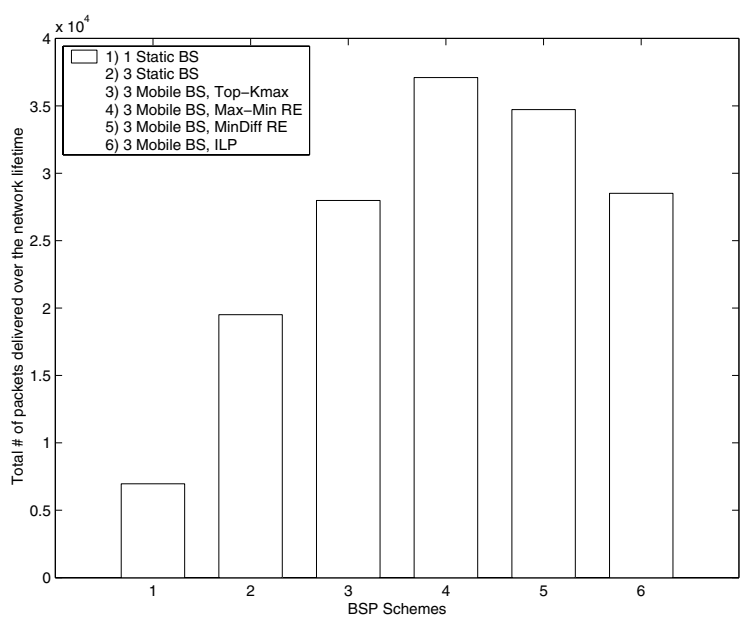

Fig. 6. Amount of packets delivered during network lifetime for different BSP algorithms. MCF routing. Initial energy at each node, E Eattery $=$ 0.05 J. One packet $=200$ bits. Range of each node, $r=10 \mathrm{~m}$.

\begin{tabular}{|l|c|c|}
\hline BSP Algorithm & $\begin{array}{c}\text { NW lifetime } \\
\text { in \# rounds } \\
(95 \% \text { confidence })\end{array}$ & $\begin{array}{c}\text { Data delivered } \\
\text { in \# packets } \\
\text { (95\% confidence) }\end{array}$ \\
\hline 1 BS & $28 \pm 0.009$ & $0.7 \times 10^{4} \pm 0.34$ \\
\hline 3 BS, static & $74 \pm 0.25$ & $1.9 \times 10^{4} \pm 14.8$ \\
\hline 3 BS, Top- $K_{\max }$ & $312 \pm 0.17$ & $2.8 \times 10^{4} \pm 1.42$ \\
\hline 3 BS, Max-Min-RE & $365 \pm 0.87$ & $3.7 \times 10^{4} \pm 42.9$ \\
\hline 3 BS, MinDiff-RE & $380 \pm 1.11$ & $3.5 \times 10^{4} \pm 45.2$ \\
\hline 3 BS, ILP & $130 \pm 0.45$ & $2.7 \times 10^{4} \pm 76.5$ \\
\hline
\end{tabular}

TABLE I

NETWORK LIFETIME AND AMOUNT OF DATA DELIVERED FOR THE VARIOUS BSP SCHEMES. MCF ROUTING.

over the 100 simulation runs as in Figs. 5 and 6). The following three interesting observations can be made from Fig. 7. 1) All the three proposed algorithms (schemes 3,4,5) utilize almost all the available network energy of $1.5 \mathrm{~J}$ before the network life ends, whereas in the other algorithms significant amount of energy is left unused even at the end of the network life, 2) although Top- $K_{\max }$ algorithm utilizes almost all the network energy, it delivers much less number of packets compared to the Max-Min-RE and MinDiff-RE algorithms; however, Top- $K_{\max }$ algorithm has the advantage of lesser complexity, and 3) Max-Min-RE and MinDiff-RE algorithms perform well in terms of total energy spent, network lifetime, and amount of data delivered, but at a higher complexity compared to Top- $K_{\max }$ algorithm and lesser complexity compared to the ILP solution.

Energy Aware Routing: All the above performance results were obtained using MCF routing and they mainly quantified the performance benefit of using multiple base stations. It is noted that MCF routing is not energy aware and network lifetime can be further increased if energy aware routing is used instead of MCF routing. We investigated the performance achieved using multiple base stations when an energy aware routing protocol that takes into account the residual energy at all nodes at the beginning of each round is employed. The energy aware routing protocol we used is similar to the one given in [4]. Table II gives the simulated performance 


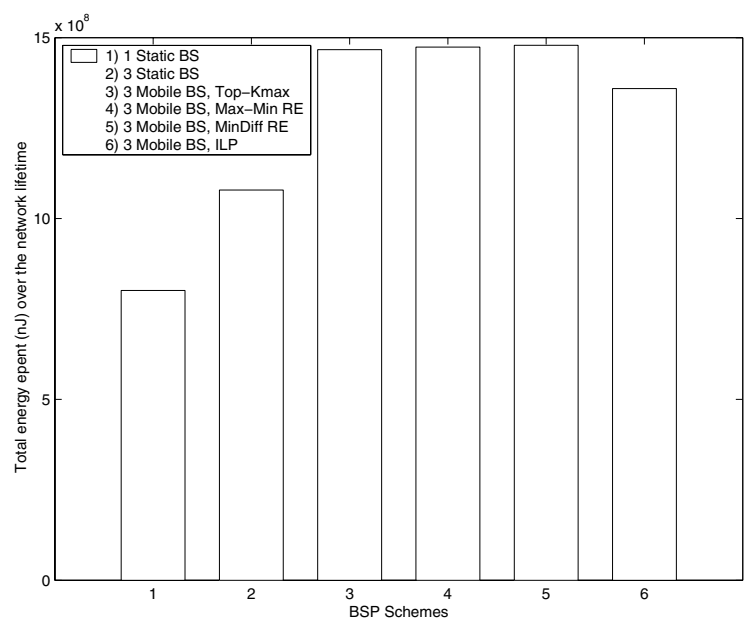

Fig. 7. Total energy spent (in $\mathrm{nJ}$ ) at the end of network life for different BSP algorithms. MCF routing. Initial energy at each node $E_{\text {battery }}=0.05 \mathrm{~J}$. One packet $=200$ bits. Range of each node, $r=10 \mathrm{~m}$.

\begin{tabular}{|l|c|c|}
\hline BSP Algorithm & $\begin{array}{c}\text { NW life time } \\
\text { in \# rounds } \\
(95 \% \text { confidence })\end{array}$ & $\begin{array}{c}\text { Data delivered } \\
\text { in \# packets } \\
(95 \% \text { confidence })\end{array}$ \\
\hline 3 BS, Mindiff-RE & $414 \pm 1.9$ & $5.3 \times 10^{4} \pm 557$ \\
\hline 3 BS, ILP & $218 \pm 2.7$ & $4.5 \times 10^{4} \pm 355$ \\
\hline
\end{tabular}

TABLE II

NETWORK LIFE TIME AND AMOUNT OF DATA DELIVERED FOR THE VARIOUS BSP SCHEMES. ENERGY AWARE ROUTING.

in terms of the network lifetime and amount of data delivered for the proposed MinDiff-RE algorithm as well as the ILP when energy aware routing is used. Comparing the results in Tables I and II, we can see that multiple base stations along with energy aware routing give increased lifetimes and amount of data delivered.

\section{CONCLUSIONS}

We proposed base stations placement algorithms to increase network lifetime and amount of data delivered during the lifetime in wireless sensor networks. We allowed multiple mobile base stations to be deployed along the periphery of the sensor network field and developed algorithms to dynamically choose the locations of these base stations so as to improve network lifetime. We proposed three energy efficient low-complexity algorithms to determine the locations of the base stations. We showed that the proposed base stations placement algorithms provide increased network lifetimes and amount of data delivered during the network lifetime compared to single base station scenario as well as multiple static base stations scenario. We also showed that the use of energy aware routing along with multiple base stations resulted in further enhancement in network lifetime.

\section{REFERENCES}

[1] W. B. Heinzelman, A. P. Chandrakasan, H. Balakrishnan, "An application-specific protocol architecture for wireless microsensor networks," IEEE Trans. Wireless Commun., vol. 1, no. 4, pp. 660-670, October 2002.
[2] A. Manjeshwar and D. P. Agrawal, "TEEN: A routing protocol for enhanced efficiency in wireless sensor networks," Proc. 15th Intl. Parallel and Distributed Processing Symposium, pp. 2009-2015, 2001.

[3] S. Lindsey and C. Raghavendra, "PEGASIS: Power-efficient gathering in sensor information systems," Proc. ICC'2001, May 2001.

[4] R. C. Shah and J. M. Rabaey, "Energy aware routing for low energy ad-hoc sensor networks" Proc. IEEE WCNC'2002, pp. 350-355, March 2002.

[5] J. Chang and L. Tassiulas, "Energy conserving routing in wireless adhoc networks," Proc. IEEE INFOCOM'00, pp. 22-31, 2000.

[6] Y. Xu, J. Heidemann, and D. Estrin, "Geography-informed energy conservation for ad-hoc routing," Proc. ACM Mobicom'01, pp. 70-84, 2001.

[7] S. R. Gandham, M. Dawande, R. Prakash, and S. Venkatesan, "Energy efficient schemes for wireless sensor networks with multiple mobile base stations, Proc. IEEE GLOBECOM2003, pp. 377-381, December 2003.

[8] K. Sohrabi, J. Gao, V. Ailawadhi, and G.J. Pottie, "Protocols for selforganization of a wireless sensor network," IEEE Pers. Commun., pp. 16-27, October 2000.

[9] F. Ye, A. Chen, S. Lu, and L. Zhang, "A scalable solution to minimum cost forwarding in large sensor networks," Proc. 10th Intl. Conf. on Comp. Commun. and Networks, pp. 304-309, 2001. 